Cahiers québécois de démographie

\title{
La morbidité hospitalière de la population de la région de Montréal : l'exemple du D.S.C. de Lakeshore
}

\section{Jaël Mongeau et Daniel Tremblay}

Volume 8, numéro 3, décembre 1979

URI : https://id.erudit.org/iderudit/600804ar

DOI : https://doi.org/10.7202/600804ar

Aller au sommaire du numéro

Éditeur(s)

Association des démographes du Québec

ISSN

0380-1721 (imprimé)

1705-1495 (numérique)

Découvrir la revue

Citer cet article

Mongeau, J. \& Tremblay, D. (1979). La morbidité hospitalière de la population de la région de Montréal : l'exemple du D.S.C. de Lakeshore. Cahiers québécois de démographie, 8(3), 187-208. https://doi.org/10.7202/600804ar
Résumé de l'article

La morbidité hospitalière est un indicateur de l'état de santé de la population. C'est toutefois un indicateur imparfait : il ne retrace que les états qui nécessitent ou qui donnent lieu à un séjour à l'hôpital, et oublie tous les cas de soins effectués dans d'autres conditions, par exemple au cabinet du médecin ou en clinique externe; il fournit un nombre d'admissions et non un nombre de personnes hospitalisées; il ne donne qu'un seul diagnostic et exclut la possibilité de diagnostics multiples.

Malgré ses imperfections, c'est un indicateur utile, en par ticulier pour étudier les différences de consommation des services hospitaliers par des populations voisines, qui ont théoriquement accès aux mêmes services.

L'intérêt de l'exploitation des données de morbidité hospitalière est ici démontré à l'aide d'un exemple, celui du territoire du Département de santé communautaire (D.S.C.) du Centre hospitalier Lakeshore General de Pointe-Claire, et de ses cinq Centres locaux de services communautaires (C.L.S.C.) existants ou prévus.

Que l'on calcule des taux globaux d'hospitalisation, en rapportant le nombre d'admissions à l'hôpital à la population, des taux par âge ou des taux par diagnostic, on constate que la population du D.S.C. Lakeshore se fait toujours moins hospitaliser que la population de la région de Montréal. Cependant, à l'intérieur du D.S.C. Lakeshore, un C.L.S.C. se distingue par des taux toujours supérieurs à ceux du D.S.C, et même de l'ensemble de la région. Or la population de ce C.L.S.C. est, entre autres, moins scolarisée, plus francophone, le pourcentage de propriétaires y est moins élevé que dans l'ensemble du D.S.C. Malgré ses limites, la donnée sur l'hospitalisation paraît donc reliée à l'état de santé comparatif des populations et à des variables socio-économiques qu'il faudra étudier davantage dans le but de mettre sur pied des programmes de prévention efficaces.
Ce document est protégé par la loi sur le droit d'auteur. L'utilisation des services d'Érudit (y compris la reproduction) est assujettie à sa politique d'utilisation que vous pouvez consulter en ligne.

https://apropos.erudit.org/fr/usagers/politique-dutilisation/ 
Cahiers québécois de démographie

Vol. 8, no 3, décembre 1979

Jaë1 MONGEAU* et Daniel TREMBLAY** : LA MORBIDITÉ HOSPITALIÉRE DE LA POPULATION DE LA RÉGION DE MONTRÉAL:

L'EXEMPLE DU D.S.C. DE LAKESHORE

(Communication présentée au 47 e congrès de l'ACFAS, section de démographie, mai 1979)

RÉSUMÉ

La morbidité hospitalière est un indicateur de l'état de santé de la population. C'est toutefois un indicateur imparfait: il ne retrace que les états qui nécessitent ou qui donnent lieu à un séjour à 1 'hôpital, et oublie tous les cas de soins effectués dans d'autres conditions, par exemple au cabinet du médecin ou en clinique externe; il fournit un nombre d'admissions et non un nombre de personnes hospitalisées; il ne donne qu'un seul diagnostic et exclut la possibilité de diagnostics multiples.

Malgré ses imperfections, c'est un indicateur utile, en particulier pour étudier les différences de consommation des services hospitaliers par des populations voisines, qui ont théoriquement accès aux mêmes services.

L'intérêt de l'exploitation des données de morbidité hospitalière est ici démontré à l'aide d'un exemple, celui du territoire du Département de santé communautaire (D.S.C.) du Centre hospitalier Lakeshore General de Pointe-Claire, et de ses cinq Centres locaux de services communautaires (C.L.S.C.) existants ou prévus.

Que l'on calcule des taux globaux d'hospitalisation, en rapportant le nombre d'admissions à l'hôpital à la population, des taux par âge ou des taux par diagnostic, on constate que la population du D.S.C. Lakeshore se fait toujours moins hospitaliser que la population de la région de Montréal. Cependant, à l'intérieur du D.S.C. Lakeshore, un C.L.S.C. se distingue par des taux toujours supérieurs à ceux du D.S.C., et même de l'ensemble de la région. Or la population de ce C.L.S.C. est, entre autres, moins scolarisée, plus francophone, le pourcentage de propriétaires y est moins élevé que dans l'ensemble du D.S.C.

Malgré ses limites, la donnée sur l'hospitalisation paraît donc reliée à l'état de santé comparatif des populations et à des variables socio-économiques qu'il faudra étudier davantage dans le but de mettre sur pied des programmes de prévention efficaces.

\footnotetext{
* I.N.R.S. - Urbanisation, 3465 Durocher, Montrêal, Québec H2X 2C6

** Département de santé communautaire, Centre hospitalier Lakeshore, 175 Stillview, suite 310, Pointe-Claire, Québec H9R 4S3
} 
Cahiers québécois de démographie

Vol. 8, no 3, décembre 1979

\title{
LA MORBIDITÉ HOSPITALIËRE DE LA
}

\section{POPULATION DE LA RÉGiON DE MONTRÉAL:}

\author{
L'EXEMPLE DU D.S.C. DE LAKESHORE
}

Par Jaël MONGEAU* et Daniel TREMBLAY**

Communication présentée au $47 e$ congrès de l'ACFAS, section de démographie, mai 1979

Introduction

Le territoire du Québec est partagé en 32 Départements de santé communautaire (D.S.C.). Leurs fonctions principales sont de planifier, de programmer et de coordonner des interventions en vue de maintenir la santé ou de prévenir la maladie de la population de leur territoire.

Pour pouvoir remplir ces fonctions, chaque D.S.C. a besoin de connaître les caractéristiques socio-démographiques et sanitaires de la

* I.N.R.S. - Urbanisation, 3465 Durocher, Montréal, Québec H2X 2C6

** Département de santé communautaire, Centre hospitalier Lakeshore, 175 Stillview, suite 310, Pointe-Claire, Québec H9R 4S3 
population qu'il dessert. Les autres intervenants du milieu, principalement les Centres locaux de services communautaires (C.L.S.C.) comptent d'ailleurs beaucoup sur les D.S.C. pour leur fournir ces données.

A premiēre vue, il peut parâtre simple d'obtenir ces données puisque des organismes gouvernementaux sont là pour les recueillir et les diffuser. Mais en fait de sérieux problèmes se sont posés pour nous. En effet, une partie des statistiques recueillies répondent à des besoins d'ordre administratif, besoins qui ne sont pas les nôtres. De plus, nous travaillons en fonction de territoires qui nous sont propres et qui ne correspondent pas aux unités géographiques avec lesquelles ces organismes fonctionnent.

Nous avons donc été forcés de nous contenter de ce qu'il y avait de mieux parmi les données disponibles et de faire des demandes spéciales pour qu'on nous les fournisse en fonction de nos territoires. Plusieurs de nos demandes n'ont pas encore été satisfaites et pourtant il y a déjà trois ans que les démarches et les négociations ont commencé. Mais en principe les 32 D.S.C. disposeront sous peu d'une banque de données. Les huit D.S.C. de la région de Montréal(1) ont décidé de les exploiter ensemble dans un dossier socio-démographique et sanitaire. Ce dernier comprendra une série de chapitres concernant des données de recensement, des données d'état civil et des données de morbidité hospitalière. Nous comptons en faire une analyse de type épidémiologique, c'est-à-dire que nous comptons décrire nos populations et établir des liens entre les différentes variables retenues, trouver des indices pertinents et très appliqués aux programmes de nos D.S.C. de façon à mieux les planifier, les programmer et les coordonner.

(1) La région socio-sanitaire du Montréal Métropolitain comprend les huit D.S.C. suivants: Lakeshore, Laval, Ma isonneuve Rosemont, Montreal General, Sacrê-Coeur, Ste-Justine, St-Luc et Verdun. 
La morbidité hospitalière comme indicateur sanitaire

La morbidité est un indicateur sanitaire qui nous rēvèle l'état de santé d'une population. En fait, il nous révẽ e son état de mauvaise santé. Mais à moins d'une enquête, on ne peut que s'en remettre à la morbidité hospitalière, c'est-à-dire aux maladies diagnostiquees lors d'une hospitalisation.

Les statistiques de morbiditê hospitalière se présentent de la façon suivante: nombre d'hospitalisations une année donnée pour telle cause de maladie(2) pour la population totale, par sexe et par groupe d'âges pour chaque D.S.C. et chaque C.L.S.C.

$$
\text { C'est un indicateur sanitaire très imparfait: }
$$

10 il ne nous donne que les maladies diagnostiquées et traitées à 1 'hôpital, laissant de côté la morbidité non ressentie ou non déclarée (un malade qui ne se sait pas malade ou qui ne se fait pas soigner) et la morbidité qui ne passe pas par l'hôpital (bureaux et cliniques de médecins, cliniques externes et urgences des hôpitaux);

$2^{0}$ il fournit le nombre d'hospitalisations et non le nombre de personnes hospitalisées;

30 il ne fournit qu'un seul diagnostic.

Nous avons pris le parti de croire qu'il est préférable d'avoir des indicateurs imparfaits dont on connaît les défauts plutôt que de ne pas en avoir du tout. En ayant un aperçu des maladies dont les gens souffrent et pour lesquelles ils consomment tant de soins hospitaliers, nous pouvons agir de façon à en prévenir l'apparition ou l'aggravation et à diminuer les hospitalisations.

(2) Classification internationale des maladies - Adaptée. 
Les caractéristiques sommaires du D.S.C. Lakeshore

Comme la recherche $n^{\prime}$ en est qu'à ses débuts, nous ne pouvons présenter ici qu'un exemple, mais il est suffisant pour démontrer l'intérêt de l'exploitation de telles données au niveau local. Il s'agit du territoire du Département de santé communautaire du centre hospitalier Lakeshore General de Pointe-Claire, ci-après nommé D.S.C. Lakeshore, et de ses cinq territoires de C.L.S.C. existants ou prévus. Le territoire du D.S.C. couvre l'ouest de l'île de Montréal. Le tableau 1 donne la liste des municipalités comprises dans chaque territoire de C.L.S.C. ainsi que 1 a population totale en 1976. On remarque que dans chaque C.L.S.C. il y a une ville plus importante que les autres du point de vue population, comme Beaconsfield dans le C.L.S.C. Sainte-Anne-de-Bellevue, ou Pierrefonds dans le C.L.S.C. du même nom. La population totale est du même ordre de grandeur partout, le C.L.S.C. le plus populeux étant celui de Lachine avec 47542 personnes et le moins populeux étant celui de Dollard-des-Ormeaux avec 36837 personnes. Ces petits effectifs invitent évidemment à la prudence quant à l'interprétation à donner aux calculs présentés ici. La population totale du D.S.C. Lakeshore s'élève à 215832 personnes, chiffre déjà plus intéressant pour l'analyse.

La morbidité hospitalière générale

Qui dit comparaison dit taux. Des taux d'hospitalisation ont été calculés, en rapportant les admissions en 1975 à la population recensée en 1976. Il peut sembler étonnant de calculer des taux avec des données pour des années différentes au numérateur et au dénominateur. Une autre série de taux a été calculée avec une estimation de la population en 1975, à titre d'essai pour certains territoires; cette estimation supposait une évolution linéaire de la population entre 1971 et 1976, le mieux que l'on puisse faire dans le contexte. Les nouveaux taux sont différents des précédents, mais ils ne changent pas fondamentalement l'ordre des territoires, d'autant plus qu'il existe des écarts importants entre les taux, comme nous le verrons plus loin. 
$\underline{\text { Tableau } 1}$

Composition territoriale et population totale en 1976 des C.L.S.C. du D.S.C. Lakeshore

\begin{tabular}{|c|c|c|}
\hline C.L.S.C. & Municipalité & Population \\
\hline 1- Pointe-Claire-Dorval & $\begin{array}{l}\text { Pointe-Claire } \\
\text { Dorval } \\
\text { Ile Dorval }\end{array}$ & $\begin{array}{r}25 \quad 917 \\
19 \quad 131 \\
\quad \quad 8 \\
45 \quad 056\end{array}$ \\
\hline 2- Sainte-Anne-de-Bellevue & $\begin{array}{l}\text { Beaconsfield } \\
\text { Kirkland } \\
\text { Baie d'Urfé } \\
\text { Sainte-Anne-de-Bellevue } \\
\text { Senneville }\end{array}$ & $\begin{array}{rr}20 & 417 \\
7 & 476 \\
3 & 955 \\
3 & 738 \\
1 & 333 \\
36 & 919\end{array}$ \\
\hline 3- Pierrefonds & $\begin{array}{l}\text { Pierrefonds } \\
\text { Roxboro } \\
\text { Saint-Raphaël-de-1 'Île-Bizard } \\
\text { Sainte-Geneviève }\end{array}$ & $\begin{array}{rr}35 & 402 \\
7 & 106 \\
4 & 101 \\
2 & 869 \\
49 & 478\end{array}$ \\
\hline 4- Dollard-des-0rmeaux & Dollard-des-Ormeaux & 36837 \\
\hline 5- Lachine & $\begin{array}{l}\text { Lachine } \\
\text { St-Pierre }\end{array}$ & $\begin{array}{r}41503 \\
6 \quad 039 \\
47 \quad 542\end{array}$ \\
\hline D.S.C. Lakeshore & & 215832 \\
\hline
\end{tabular}


Le tableau 2 présente les taux globaux d'hospitalisation pour 1e D.S.C. Lakeshore et ses C.L.S.C. Pour des fins de comparaison, les mêmes taux ont aussi été calculés pour la région de Montréal et l'ensemble du Québec. La région de Montréal comprend ici l'île de Montréal et l'île Jésus, et c'est l'ensemble du territoire couvert par cette recherche.

\section{Tableau 2}

Taux d'hospitalisation (\%) de la population totale, D.S.C. Lakeshore par C.L.S.C.. région de Montréal et ensemble du Québec, 1975

\begin{tabular}{|l|c|}
\hline \multicolumn{1}{|c|}{ Territoire } & $\begin{array}{c}\text { Taux d'hospita- } \\
\text { 1isation (\%) }\end{array}$ \\
\hline 1- C.L.S.C. Pointe-Claire-Dorval & 10,5 \\
2- C.L.S.C. Sainte-Anne-de-Bellevue & 9,2 \\
3- C.L.S.C. Pierrefonds & 10,0 \\
4- C.L.S.C. Dollard-des-Ormeaux & 10,1 \\
5- C.L.S.C. Lachine & 15,1 \\
D.S.C. Lakeshore & 11,1 \\
Région de Montréal & 12,4 \\
\hline
\end{tabular}

Dans l'ensemble du Québec, les admissions à 1'hôpital en 1975 représentent $14 \%$ de 1 a population, ce qui peut sembler énorme. Il ne faut surtout pas oublier que les données concernent le nombre d'admissions et non le nombre de personnes: la même personne peut être comptée plus d'une fois, si elle a fait plus d'un séjour à l'hôpital la même année. De plus 
les naissances sont aussi comptées, en plus des hospitalisations pour accouchement; donc à chaque naissance à 1'hôpital correspond au moins deux séjours à 1'hôpital: celui de la mère et celui de l'enfant. Nous verrons à exclure ces catégories dans d'autres calculs. Le taux global d'hospitalisation est moins élevé dans la région de Montréal, et moins encore pour le D.S.C. Lakeshore. Le taux est assez semblable pour tous les C.L.S.C. du D.S.C. Lakeshore, sauf pour celui de Lachine, encore plus élevé que dans l'ensemble du Québec.

La première chose qu'on est tenté de vérifier, c'est si ces différences sont dues à des différences de structure par âge. Comme on a la chance d'avoir des données par grand groupe d'âges selon le sexe, il est possible de calculer des taux par groupe d'âges. Ces taux sont illustrés au graphique 1, pour 1'ensemble du Québec, la région de Montréal, 1e D.S.C. Lakeshore et 1e C.L.S.C. de Lachine, celui qui a un taux global supérieur à celui du Québec.

Pour le sexe masculin, le même scénario se rêpète à chaque groupe d'âges, sauf pour le C.L.S.C. de Lachine: les taux du Québec sont supérieurs à ceux de la région et ces derniers sont supérieurs à ceux du Lakeshore. Les taux du C.L.S.C. de Lachine sont toujours supérieurs à ceux de la région, mais ils sont inférieurs à ceux du Québec à partir de 50 ans; or la population masculine de 50 ans et plus représente $24,5 \%$ de la population masculine du C.L.S.C. Lachine, et $19,5 \%$ de celle de 1 'ensemble du québec. Si le taux global du C.L.S.C. Lachine est supérieur à ce1 ui de l'ensemble du Québec, c'est en partie parce que 1a population y est plus vieille. Cependant, la morbidité hospitalière du C.L.S.C. Lachine reste plus importante à chaque groupe d'âges que dans l'ensemble de son D.S.C. et de sa région. A noter que les courbes du sexe masculin ressemblent à des courbes de mortalité par âge: la morbidité est très forte à 0-4 ans, puis elle atteint son minimum au groupe suivant, pour ensuite augmenter continuellement avec l'âge. 
TAUX D'HOSPITALISATION PAR GROUPE D'AGES SELON LE SEXE, 1975.

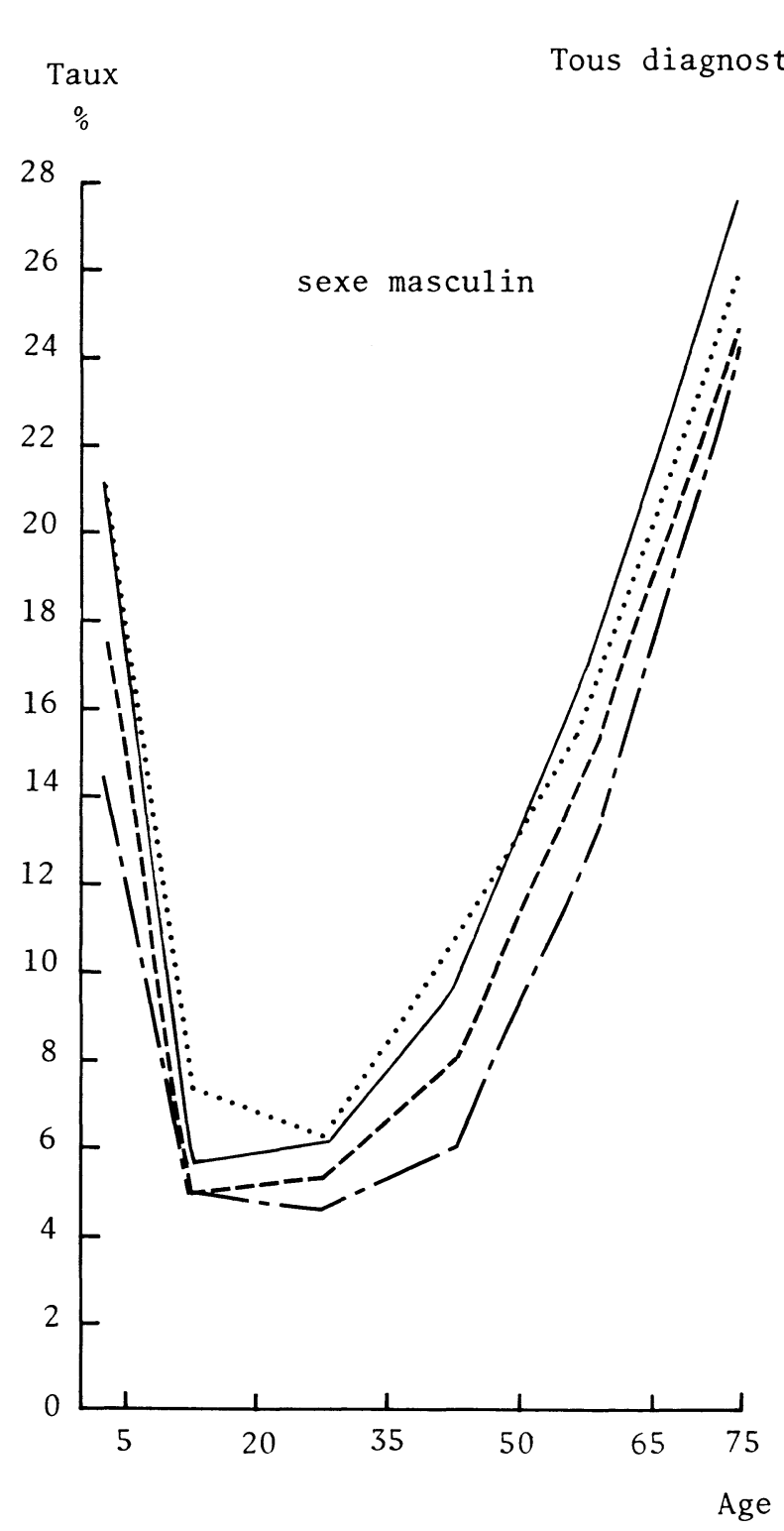

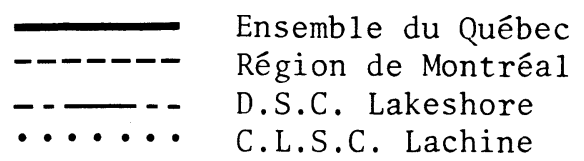
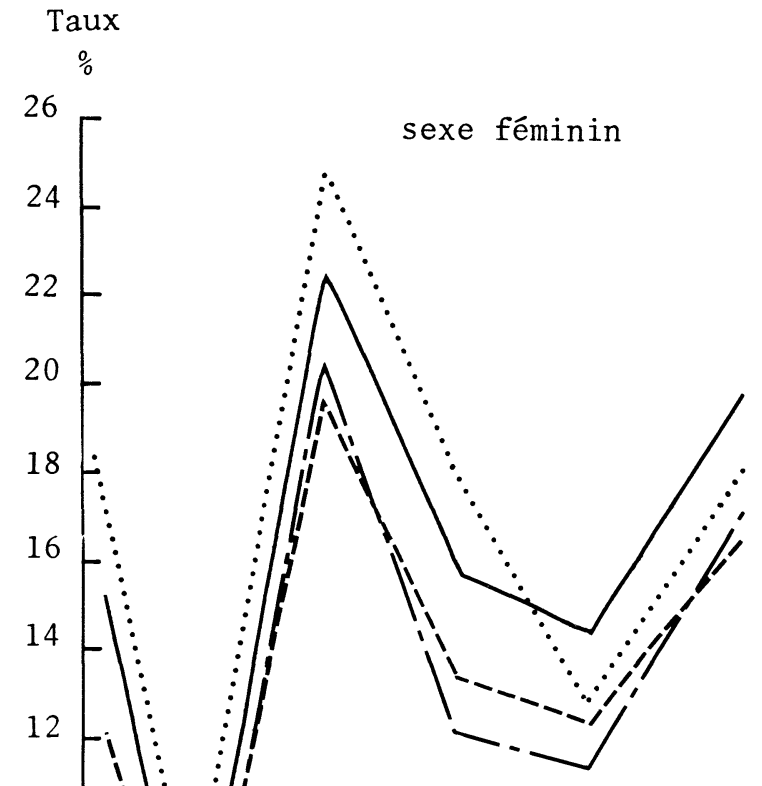

10
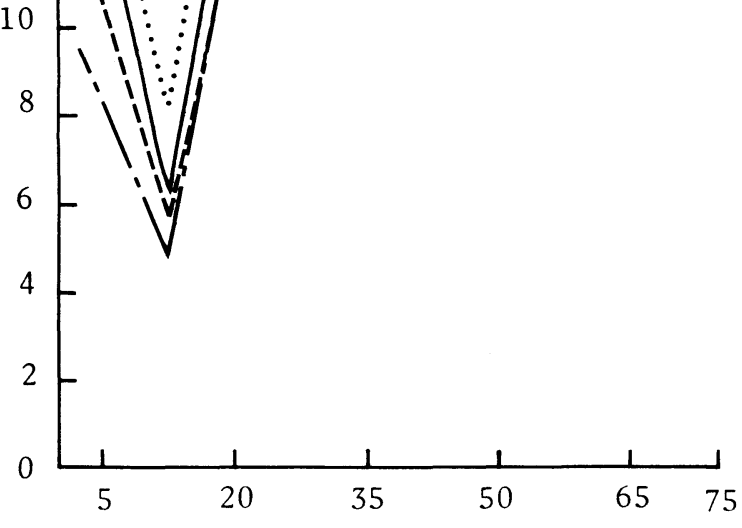

Age

Tous diagnostics

(sauf naissances et

hospitalisations

liées à 1a grossesse)

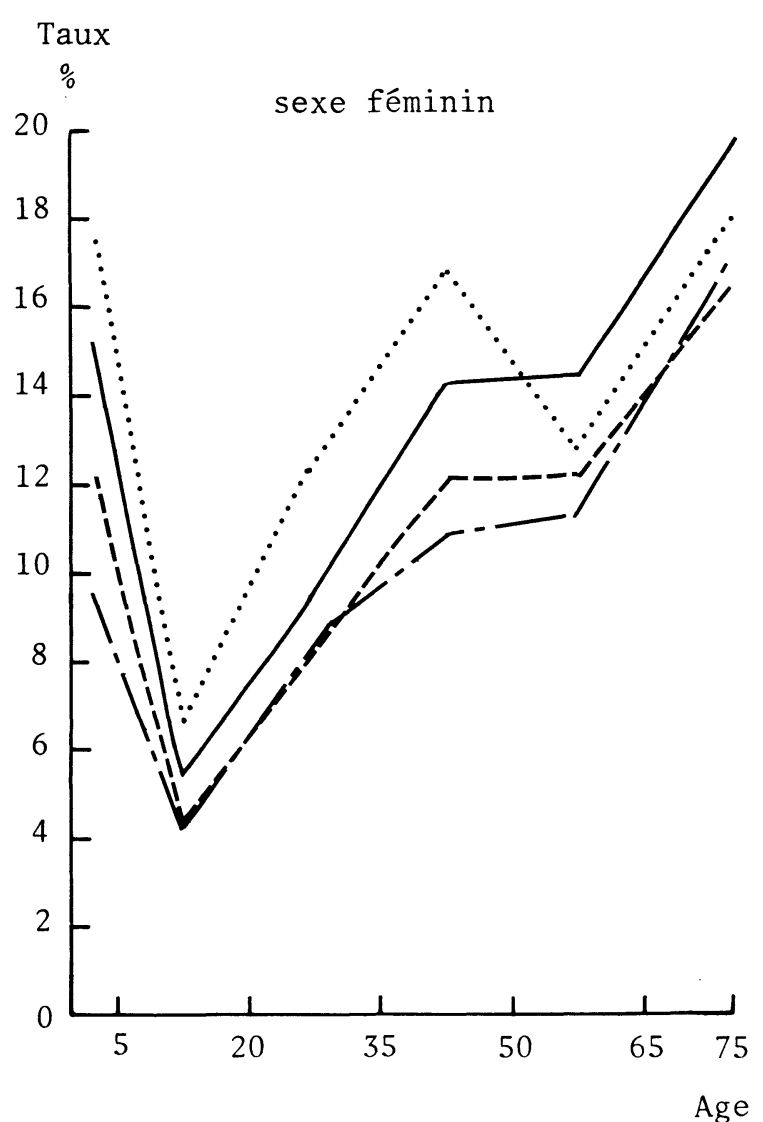


GRAPHIQUE 2

TAUX D'HOSPITALISATION SELON LE DIAGNOSTIC, 1975.

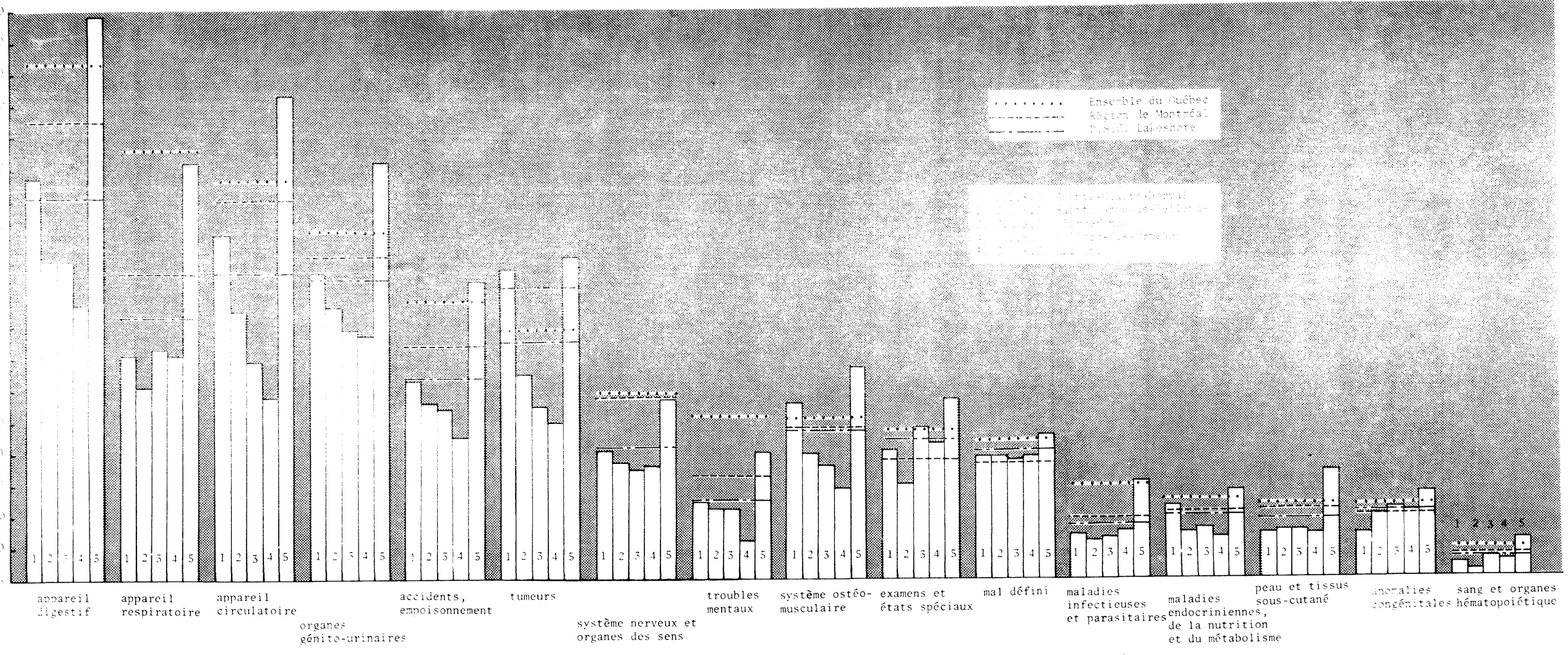


La comparaison par territoire des taux féminins est assez semblable à celle des taux masculins, bien que les taux eux-mêmes soient notablement différents. Même en enlevant les hospitalisations reliées à la grossesse, à la droite du graphique 1, les taux du sexe féminin ne subissent pas une augmentation continue à partir de 5-19 ans comme ceux du sexe masculin. On observe un palier entre 40 et 55 ans, et les taux aux deux extrêmes sont moins élevés que ceux du sexe masculin: seuls les taux à 5-19 ans et à 50-64 ans sont assez semblables pour les deux sexes.

\section{Les hospitalisations selon le diagnostic}

Après avoir vérifié que les différences entre les taux globaux d'hospitalisation ne s'expliquent pas uniquement par des différences de structure par âge, on peut se demander si les différences vont dans le même sens pour tous les diagnostics. Les admissions sont divisées en 99 groupes de diagnostics réunis en 19 grands groupes.

Le graphique 2 illustre les taux d'hospitalisation de 16 des 19 grands groupes, pour 1'ensemble du Québec, la région de Montréal, le D.S.C. Lakeshore et ses cinq C.L.S.C. Quand on compare le D.S.C., la région et l'ensemble du Québec, les taux sont dans le même ordre que le taux global pour 12 diagnostics sur 16. Le taux du Québec est le plus élevé partout, sauf pour le diagnostic "tumeurs", alors qu'il est dépassé par celui de la région de Montréal. Le taux de la région de Montréal est entre celui du Québec et celui du D.S.C. Lakeshore, sauf pour trois diagnostics: examens et états spéciaux, symptômes et états morbides mal définis et anomalies congénitales, alors que le taux du D.S.C. lui est supérieur.

Quand on regarde les taux par C.L.S.C., on voit surtout dépasser les taux du C.L.S.C. de Lachine (5) pour tous les diagnostics, mais on voit aussi que les taux du C.L.S.C. Pointe-Claire-Dorval (1) se classent souvent bons deuxièmes, alors que les taux du C.L.S.C. Dollarddes-Ormeaux (4) sont les plus bas pour la moitié des diagnostics. Les 
taux varient au moins du simple au double entre le plus élevé et le plus bas taux à l'intérieur du même diagnostic, sauf pour les maladies des organes génito-urinaires, les maladies du système nerveux et des organes des sens et les symptômes et états morbides mal définis, alors qu'ils varient un peu moins. Les C.L.S.C. Sainte-Anne-de-Bellevue, Pierrefonds et Dollard-des-0rmeaux ont finalement des taux assez semblables.

Au graphique 2, les diagnostics sont présentés par ordre de leur importance numérique dans 1'ensemble du Québec; par exemple, parmi les 16 diagnostics illustrés, ce sont les maladies de l'appareil digestif qui sont responsables du plus grand nombre d'hospitalisations en 1975 au Québec. Il est toutefois primordial de signaler ici que parmi les trois groupes de diagnostics non illustrés figure celui des diagnostics reliés à la grossesse (grossesse, accouchement et suites de couches), qui se classe avant les maladies de l'appareil digestif, donc au premier rang quant au nombre d'hospitalisations au Québec. Ce groupe de diagnostics n'a pas été illustré au graphique 2, parce que son importance dépend à la fois de la fécondité et de l'état de santé des femmes. Les deux autres groupes omis, c'est-à-dire les enfants nés vivants et les causes de morbidité et de mortalité périnatales, l'ont été parce que leur importance dans l'ensemble de la population dépend aussi de la féconditê.

En excluant les diagnostics reliês à la grossesse, les six principaux groupes de diagnostics pour lesquels la population du Québec a été hospitalisée en 1975 sont, dans l'ordre, les maladies de 1'appareil digestif (cirrhose du foie, ulcères d'estomac, gastro-entérite, ...), celles de 1'appareil respiratoire (bronchite, pneumonie,...), celles de l'appareil circulatoire (maladies du coeur, ...), celles des organes génito-urinaires, les accidents (fractures, empoisonnements, traumatismes, ...) et les tumeurs. Plus localement, ce sont encore les mêmes groupes qui sont en tête: au tableau 3 qui donne le rang des principaux diagnostics, on voit que les six mêmes groupes de diagnostics occupent toujours les six premiers rangs. Les diagnostics ne sont cependant pas toujours dans le même ordre: si les maladies de l'appareil digestif occupent 
Rang des six principaux diagnostics par territoire (en excluant les diagnostics reliés à la grossesse et les enfants nés vivants), 1975

\begin{tabular}{|c|c|c|c|c|c|c|c|c|}
\hline $\begin{array}{c}\text { Groupe de } \\
\text { diagnostics }\end{array}$ & $\begin{array}{l}0 \\
\otimes \\
\frac{0}{10} \\
2\end{array}$ & 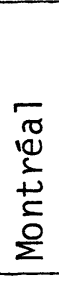 & 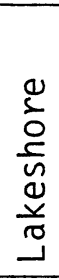 & 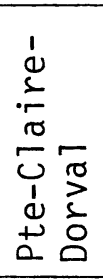 & 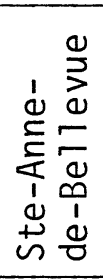 & $\begin{array}{l}0 \\
0 \\
0 \\
0 \\
4 \\
0 \\
2 \\
\frac{1}{2} \\
\frac{1}{2} \\
\end{array}$ & 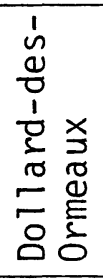 & 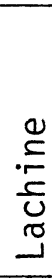 \\
\hline $\begin{array}{l}\text { Maladies de l'appareil } \\
\text { digestif }\end{array}$ & 1 & 1 & 1 & 1 & 1 & 1 & 1 & 1 \\
\hline $\begin{array}{l}\text { Maladies de l'appareil } \\
\text { respiratoire }\end{array}$ & 2 & 4 & 4 & 5 & 5 & 3 & 3 & 4 \\
\hline $\begin{array}{l}\text { Maladies de l'appareil } \\
\text { circulatoire }\end{array}$ & 3 & 2 & 2 & 2 & 3 & 4 & 4 & 2 \\
\hline $\begin{array}{l}\text { Maladies des organes } \\
\text { génito-urinaires }\end{array}$ & 4 & 3 & 3 & 4 & 2 & 2 & 2 & 3 \\
\hline $\begin{array}{l}\text { Accidents, empoisonnements } \\
\text { et traumatismes }\end{array}$ & 5 & 6 & 6 & 6 & 6 & 6 & 6 & 6 \\
\hline Tumeurs & 6 & 5 & 5 & 3 & 4 & 5 & 5 & 5 \\
\hline
\end{tabular}


toujours le premier rang, les maladies de l'appareil respiratoire ne sont au deuxième rang que pour l'ensemble du Québec; elles sont même au cinquième rang dans deux territoires de C.L.S.C. Dans la région de Montréal, 1e D.S.C. Lakeshore et ses C.L.S.C., les maladies de 1'apparei1 respiratoire sont partout dépassées par les maladies des organes génitourinaires. Ces dernières ne sont pourtant pas plus importantes dans la région que dans l'ensemble du Québec, comme 1'indique le graphique 2.

On peut être tenté de rechercher, à l'intērieur d'un groupe de diagnostics, quels diagnostics plus spécifiques amènent un séjour à 1 'hôpital, ou sont responsables des différences entre territoires. Le tableau 4 détaille, à titre d'exemple, les taux du groupe des maladies de l'appareil digestif en quelques sous-groupes. Le tiers environ des hospitalisations de ce groupe sont le fait des maladies du foie, de la vésicule et du pancréas, quels que soient le territoire et le taux global du groupe. Par ailleurs, le tableau 4 nous apprend que malgré des variations du simple au double au total (taux de 88 pour 1e C.L.S.C. Dollard-des-Ormeaux et de 179 pour celui de Lachine), un diagnostic très précis semble affecter la population de façon égale partout: il s'agit de l'appendicite.

Les hospitalisations par suite d'accident

Un groupe de diagnostics retient particulièrement 1'attention parce qu'il se prête admirablement bien à la prévention: il s'agit des accidents. Les hospitalisations pour accident sont détaillées selon le diagnostic (fracture, brûlure, empoisonnement, etc.), mais aussi selon le lieu de l'accident. Sont distingués six types d'accidents: de la circulation, de la ferme, du travail, accidents à domicile, au jeu et autres, Te résidu comprenant les accidents survenus dans les édifices publics. On peut ignorer les hospitalisations pour accident de la ferme, au nombre de 42 pour la région de Montréal, et de 6 pour le D.S.C. Lakeshore en 1975. Les autres types d'accidents sont illustrés au graphique 3. Dans l'ensemble du Québec, il y a autant d'hospitalisations 
Taux d'hospitalisation (pour 10000 ) par sous-groupe de diagnostics pour le groupe des maladies de l'appareil digestif, 1975

\begin{tabular}{|c|c|c|c|c|c|c|c|c|}
\hline $\begin{array}{l}\text { Sous-groupe } \\
\text { de diagnostics }\end{array}$ & 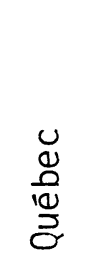 & 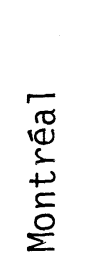 & $\begin{array}{l}0 \\
\vdots \\
0 \\
\frac{0}{n} \\
0 \\
\frac{1}{0}\end{array}$ & 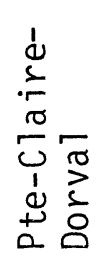 & 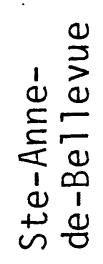 & $\begin{array}{l}0 \\
0 \\
\frac{0}{0} \\
4 \\
0 \\
\frac{1}{2} \\
\frac{1}{0} \\
-\frac{1}{2}\end{array}$ & 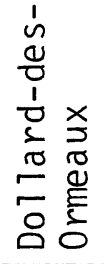 & 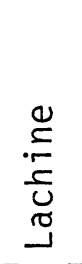 \\
\hline $\begin{array}{l}\text { Cavité buccale, glandes } \\
\text { salivaires et maxillaires }\end{array}$ & 20 & 7 & 9 & 7 & 6 & 5 & 4 & 19 \\
\hline $\begin{array}{l}\text { Maladies de } 1 \text { 'oesophage, de } \\
1 \text { 'estomac et du duodénum }\end{array}$ & 24 & 18 & 13 & 15 & 10 & 10 & 6 & 21 \\
\hline Appendicite & 13 & 12 & 12 & 12 & 13 & 11 & 11 & 12 \\
\hline Hernie abdominale & 33 & 35 & 31 & 32 & $? 7$ & 26 & 27 & 42 \\
\hline $\begin{array}{l}\text { Autres maladies de l'intes- } \\
\text { tin et du péritoine }\end{array}$ & 21 & 19 & 16 & 16 & 12 & 11 & 14 & 26 \\
\hline $\begin{array}{l}\text { Maladies du foie, de la } \\
\text { vésicule et du pancréas }\end{array}$ & 53 & 53 & 41 & 45 & 32 & 37 & 26 & 59 \\
\hline Total & 164 & 144 & 122 & 127 & 100 & 100 & 88 & 179 \\
\hline
\end{tabular}


p. 10,000 TAUX D'HOSPITALISATION POUR ACCIDENTS SELON LE LIEU DE L'ACCIDENT, 1975.

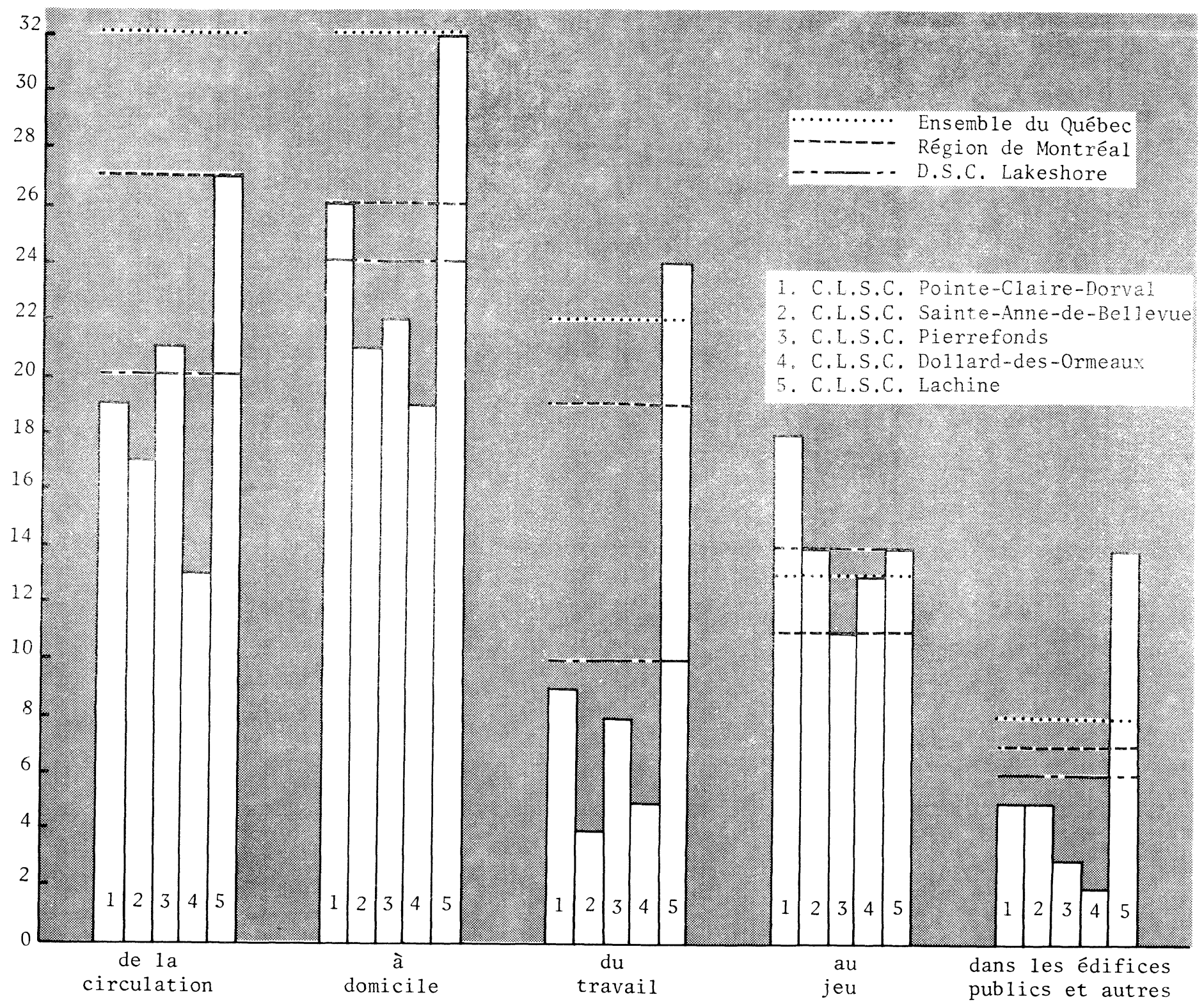


pour accidents à domicile que pour accident de la circulation. Dans la région de Montréal, 11 y a plus d'hospitalisations pour accidents de la circulation que pour accidents à domicile, alors que c'est le contraire pour le D.S.C. Lakeshore et chacun de ses C.L.S.C. Les accidents du travail viennent en troisième pour l'ensemble du Québec, la région de Montréal et le C.L.S.C. Lachine, mais sont moins importants que les accidents au jeu dans les autres territoires. Pour tous les types d'accidents sauf les accidents au jeu, les taux d'hospitalisation sont plus élevés dans l'ensemble du Québec que dans la région de Montréal, et plus élevés dans la région de Montréal que pour le D.S.C. Lakeshore. Pour les accidents au jeu, le taux du D.S.C. Lakeshore est plus élevé que celui de l'ensemble du Québec, en même temps que le taux du C.L.S.C. Pointe-Claire-Dorval est singulièrement le plus élevé parmi les C.L.S.C.; pour les autres types d'accidents, c'est 1e C.L.S.C. Lachine qui se classe en tête des C.L.S.C.

C'est pour les accidents de la circulation et du travail qu'il y a le plus de différences entre les taux du Québec, de Montréal et du D.S.C. Lakeshore. C'est pourquoi les deux types d'accidents sont illustrés par groupe d'âges aux graphiques 4 et 5 . Tout d'abord les deux types d'accidents affectent plus le sexe masculin que le sexe féminin. Quel que soit le territoire ou le sexe, c'est entre 15 et 25 ans que 1 es taux sont les plus élevés pour les accidents de la circulation. C'est aussi à partir de 15 ans que commencent à se manifester les différences entre les territoires: entre 15 et 25 ans pour le sexe masculin, les taux du Québec sont nettement plus élevés que ceux des deux autres territoires, alors qu'après 25 ans ils sont assez semblables à ceux de 1 a région de Montréa1. Ce sont alors les taux du D.S.C. qui sont nettement inférieurs à ceux des deux autres territoires.

Les accidents du travail touchent une minorité de femmes. Du côté du sexe masculin, tous les âges sont touchés également entre 20 et 65 ans dans 1e D.S.C. Lakeshore, alors que les taux augmentent jusqu'à atteindre un sommet à 35-49 ans dans l'ensemble du Québec et la région 
GRAPHIQUE 4

TAUX D'HOSPITALISATION PAR GROUPE D'AGES SELON LE SEXE, ACCIDENTS DE LA CIRCULATION, 1975.
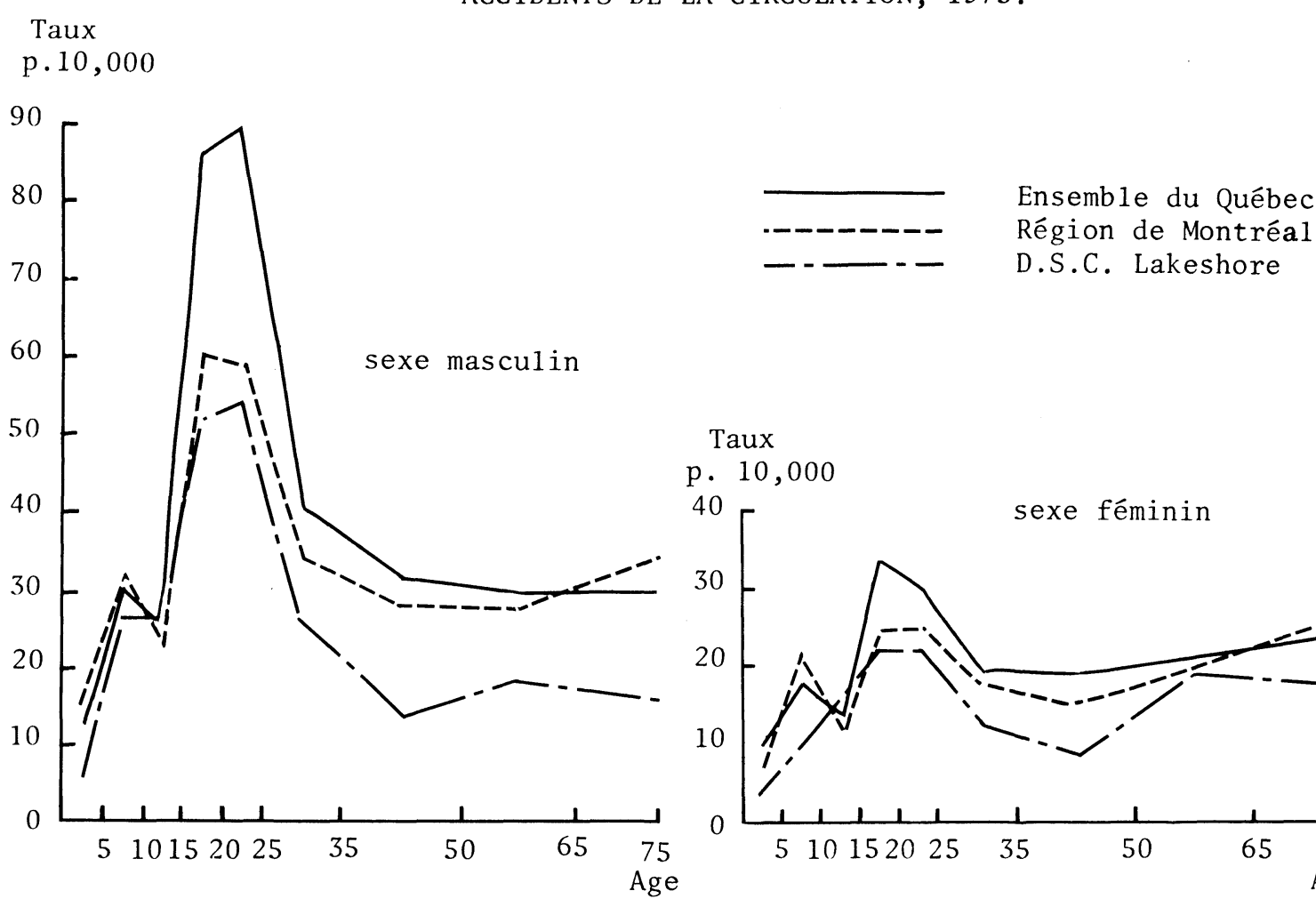

Taux

p. 10,000

40 sexe féminin

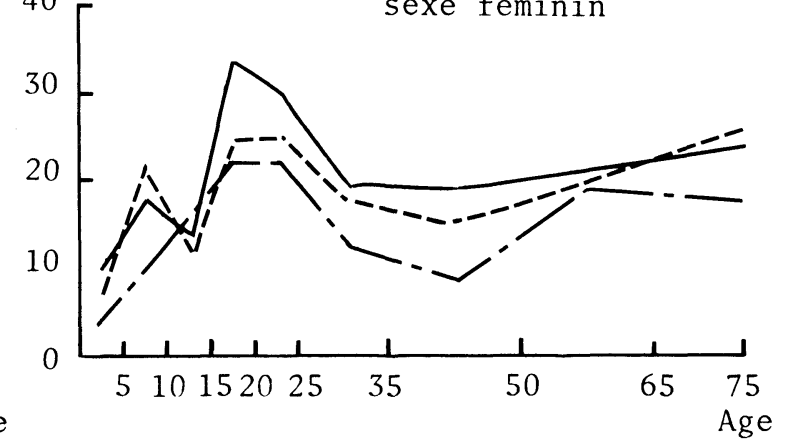

Taux

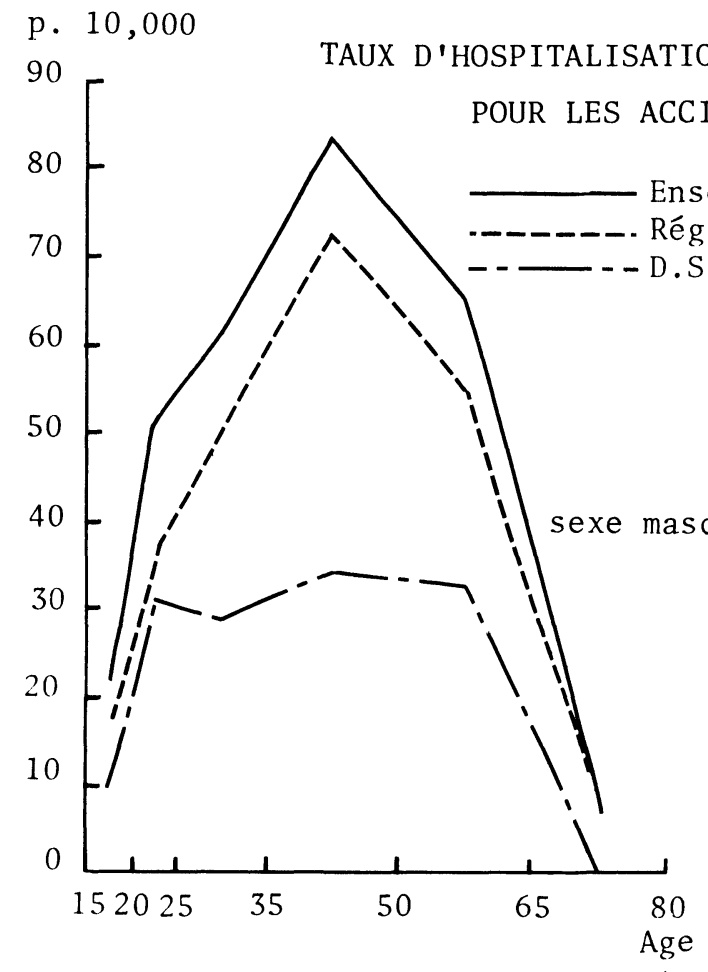

GRAPHIQUE 5

sexe féminin

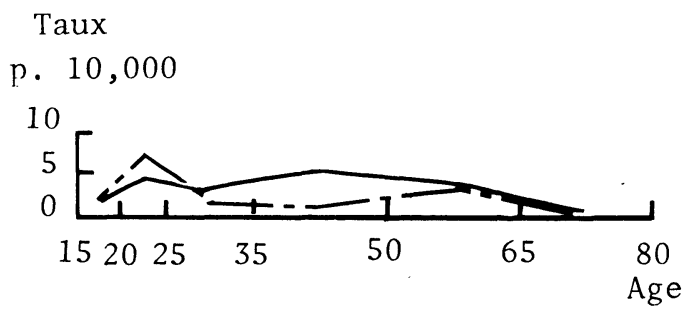


de Montréal, pour ensuite diminuer. Les différences auraient sans doute intérêt à être rapprochées des différences dans la structure professionnelle de la population selon le territoire.

\section{Conclusion}

De façon générale, ces quelques tableaux et graphiques nous ont appris que la population du D.S.C. Lakeshore s'était fait beaucoup moins hospitaliser en 1975 que l'ensemble de la population montréalaise, et cette dernière moins que l'ensemble de la population québécoise. Cependant, à l'intérieur du D.S.C. Lakeshore, un C.L.S.C. se distingue par des taux d'hospitalisation souvent supérieurs à ceux de l'ensemble du Québec. Ces constatations valent pour presque tous les diagnostics, les groupes d'âges, les sexes et les types d'accidents.

Sans vouloir véritablement rechercher les causes de ces différences, on peut être tenté de faire des rapprochements avec des données socio-économiques sur la population des territoires étudiés. Alors que 1a population n'ayant pas atteint la ge année représente $14,7 \%$ de 1a population de 15 ans et plus pour le D.S.C. Lakeshore en 1976, elle représente $30,2 \%$ dans 1e C.L.S.C. Lachine, 5,5\% dans 1e C.L.S.C. Do11ard-desOrmeaux et $33 \%$ dans l'ensemble du Québec. Les propriétaires sont au nombre de $30,4 \%$ dans 1e C.L.S.C. Lachine, alors qu'ils sont plus de 60\% dans chacun des autres territoires de C.L.S.C. du D.S.C. Lakeshore. II y a 59\% de francophones dans le territoire du C.L.S.C. Lachine, contre $33,1 \%$ en moyenne dans 1e D.S.C.

Il semblerait bel et bien que, malgré ses limites, la donnée sur l'hospitalisation nous apprenne quelque chose sur l'état de santé comparatif des populations, à moins que ce soit sur les différences d'habitudes de vie de ces mêmes populations. Par exemple, toutes les populations ne semblent pas également soumises au risque d'avoir un accident de travail ou de jeu nécessitant 1'hospitalisation. Or changer les habitudes de vie ne requiert pas les mêmes moyens d'action que chan- 
ger l'état de santé et c'est pourquoi il est important de tenter d'identifier le plus possible les causes des différences dans les taux d'hospitalisation, notamment pour un même diagnostic. La présente recherche ne pourra malheureusement aller très loin dans cette voie, vu qu'elle ne peut puiser d'éléments d'explication des différences que dans les données du recensement, mais elle permettra, nous l'espérons, de faire ressortir les populations particulières au sujet desquelles il faudra sans doute faire des recherches plus approfondies. 


\section{RÉFÉRENCES BIBL IOGRAPHIQUES}

Bédard, Robert, Etude sommaire de la situation de l'information statistique sur la société québécoise, Conseil des Affaires sociales et de la famille, avrit 1977.

Emond, Aline, Gérald Lescarbeault et Gaël 01 ivier, Population lavalloise: certaines caractéristiques socio-démographiques, D.S.C. Cité de la santé, Laval, première édition, août 1978.

Mausner, Judith S. et Anita K. Bahn, Epidemiology, and Introductory Text, W.B. Saunders Company, Philadelphia, 1974.

McWhinnie, John R., Barbara L. Ouellet et Jean-Marie Lance, Indicateurs du domaine de la santé, Canada et Provinces, Direction générale de la planification à long terme (santé), Ministère de la Santé nationale et du Bien-être social, Ottawa, décembre 1976.

Phisel, Rosel ine et Robert Charbonneau, Rapport sur la morbidité du D.S.C. Montréal General, Montréal, août 1977.

Sanders, Barkev S., "Measuring Community Health Levels", dans American Journal of Public Health, vol. 54, no 7, July 1964, p. 1063-1070.

Susser, M.W., "Social Science and Public Health", dans Hobson, W., The Theory and Practice of Public Health, Oxford University Press, London, 1965. 